Pacific Journal of Mathematic 


\title{
SOME THEOREMS ON GENERALIZED DEDEKIND SUMS
}

\author{
L. CARLitz
}

1. Introduction. Using a method developed by Rademacher [5], Apostol [1] has proved a transformation formula for the function

$$
G_{p}(x)=\sum_{m, n=1}^{\infty} n^{-p} x^{m n} \quad(|x|<1)
$$

where $p$ is a fixed odd integer $>1$. The formula involves the coefficients

$$
c_{r}(h, k)=\sum_{\mu(\bmod k)} P_{p+1-r}\left(\frac{\mu}{k}\right) P_{r}\left(\frac{h \mu}{k}\right) \quad(0 \leq r \leq p+1),
$$

where $(h, k)=1$, the summation is over a complete residue system $(\bmod k)$, and $P_{r}(x)=\bar{B}_{r}(x)$, the Bernoulli function.

We shall show in this note that the transformation formula for (1.1) implies a reciprocity relation involving $c_{r}(h, k)$, which for $r=p$ reduces to Apostol's reciprocity theorem [1, Th. $1 ; 2$, Th. 2] for the generalized Dedekind sum $c_{p}(h, k)$. In addition, we prove some formulas for $c_{r}(h, k)$ which generalize certain results proved by Rademacher and Whiteman [6]. Finally we derive a representation of $c_{r}(h, k)$ in terms of so-called "Eulerian numbers".

2. Some preliminaries. It will be convenient to recall some properties of the Bernoulli function $P_{r}(x)$; by definition, $P_{r}(x)=B_{r}(x)$ for $0 \leq x<1$, and $P_{r}(x+1)=P_{r}(x)$. Also we have the formulas

$$
\sum_{r=0}^{k-1} P_{r}\left(t+\frac{r}{k}\right)=k^{1-m} P_{r}(k t), \quad P_{r}(-x)=(-1)^{r} P_{r}(x)
$$

It follows from the second of $(2.1)$ that $c_{r}(h, k)=0$ for $p$ even and $0 \leq r \leq p+1$. We have also 


$$
c_{0}(h, k)=c_{p+1}(h, k)=k^{-p} B_{p+1}
$$

provided $(h, k)=1$. Further, it is clear from the second of $(2.1)$ that

$$
c_{r}(-h, k)=(-1)^{r} c_{r}(h, k) \text {. }
$$

Now as in $[5,321]$ put $x=e^{2 \pi i \tau}$,

$$
\tau=\frac{i z+h}{k}, \tau^{\prime}=\frac{i z^{-1}+h^{\prime}}{k}
$$

so that, on eliminating $z$, we get

$$
\tau^{\prime}=\frac{h^{\circ} \tau+k^{\circ}}{k \tau-h} \quad\left(h h^{\circ}+k k^{\circ}+1=0\right)
$$

thus (2.4) is a unimodular transformation. Now Apostol's transformation formula $[1$, Th. 2] reads (in our notation)

$$
\begin{aligned}
G_{p}\left(e^{2 \pi i \tau}\right) & =(i z)^{p-1} G_{p}\left(e^{2 \pi i \tau^{\prime}}\right)-\frac{1}{2}\left(\frac{2 \pi z}{k}\right)^{p} \frac{B_{p+1}}{(p+1) !} \\
& +\frac{i^{p-1}}{2 z}\left(\frac{2 \pi}{k}\right)^{p} \frac{B_{p+1}}{(p+1) !}+\frac{(2 \pi i)^{p}}{2 \cdot p !} c_{p}(h, k) \\
& +\frac{(2 \pi)^{p} z^{p-1}}{2(p+1) !} \sum_{r=0}^{p-2}\left(\begin{array}{c}
p+1 \\
r+1
\end{array}\right) e^{\pi i(r-1) / 2} z^{-r} \sum_{\mu=1}^{k} P_{p-r}\left(\frac{h^{\prime} \mu}{k}\right) P_{r+1}\left(\frac{\mu}{k}\right) .
\end{aligned}
$$

Making use of (1.2), (2.2), and (2.3), we easily verify that this result can be put in the form

$$
G_{p}\left(e^{2 \pi i \tau}\right)=(k \tau-h)^{p-1} G_{p}\left(e^{2 \pi i \tau^{\prime}}\right)+\frac{(2 \pi i)^{p}}{2(p+1) !} f(h, k ; \tau)
$$

where

$$
f(h, k ; \tau)=\sum_{r=0}^{p+1}\left(\begin{array}{c}
p+1 \\
r
\end{array}\right)(k \tau-h)^{p-r} c_{r}(h, k) .
$$

We remark that $(2.6)$ can be written in the symbolic form 


$$
(k \tau-h) f(h, k ; \tau)=(k \tau-h+c(h, k))^{p+1},
$$

where it is understood that after expanding the right member of $(2.7)$ by the binomial theorem, $c^{r}(h, k)$ is replaced by $c_{r}(h, k)$.

We shall require an explicit formula for $f(0,1 ; \tau)$. Since, by $(1.2)$,

$$
c_{r}(0,1)=P_{p+1-r}(0) P_{r}(0)=B_{p+1-r} B_{r}
$$

it is clear that $(2.6)$ implies

$$
f(0,1 ; \tau)=\frac{1}{\tau} \sum_{r=0}^{p+1}\left(\begin{array}{c}
p+1 \\
r
\end{array}\right) B_{p+1-r} B_{r} \tau^{p+1-r}=\frac{1}{\tau}(B+\tau B)^{p+1} .
$$

If in (2.4) we replace $\tau$ by $-1 / \tau$, then $\tau^{\prime}$ becomes

$$
\tau^{*}=\frac{-k^{\bullet} \tau+h^{\bullet}}{h \tau+k}
$$

and $(2.5)$ becomes

$$
G_{p}\left(e^{-2 \pi i / \tau}\right)=\left(\frac{h \tau+k}{\tau}\right)^{p-1} G_{p}\left(e^{2 \pi i \tau^{*}}\right)+\frac{(2 \pi i)^{p}}{2(p+1) !} f\left(h, k ;-\frac{1}{\tau}\right) .
$$

By (2.5) and (2.8) we have

$$
G_{p}\left(e^{2 \pi i \tau}\right)=\tau^{p-1} G_{p}\left(e^{-2 \pi i / \tau}\right)+\frac{(2 \pi i)^{p}}{2 \tau(p+1) !}(B+\tau B)^{p+1}
$$

and by (2.5) and (2.9),

$$
G_{p}\left(e^{2 \pi i \tau}\right)=(h \tau+k)^{p-1} G_{p}\left(e^{2 \pi i \tau *}\right)+\frac{2 \pi i}{2(p+1) !} f(-k, h ; \tau) .
$$

Comparison of (2.10), (2.11), (2.12) yields

$$
f(-k, h ; \tau)=\tau^{p-1} f\left(h, k ;-\frac{1}{\tau}\right)+\frac{1}{\tau}(B+\tau B)^{p+1},
$$

or with $\tau$ replaced by $-1 / \tau$, 


$$
f(h, k ; \tau)=\tau^{p-1} f\left(-k, h ;-\frac{1}{\tau}\right)+\frac{1}{\tau}(B+\tau B)^{p+1}
$$

(For the above, compare [3, pp. 162-163]).

3. The main results. In (2.7) replace $h, k, \tau$ by $-k, h,-1 / \tau$ respectively; we get

$$
\frac{k \tau-h}{\tau} f\left(-k, h ;-\frac{1}{\tau}\right)=\left(\frac{k \tau-h}{\tau}+c(-k, h)\right)^{p+1}
$$

By $(2.3)$, it is clear that $(2.13)$ becomes

$$
\begin{aligned}
\tau(k \tau-h+c(h, k))^{p+1} \\
\quad=(\tau c(k, h)-\tau k+h)^{p+1}+(k \tau-h)(B+\tau B)^{p+1}
\end{aligned}
$$

Comparison of the coefficients of $\tau^{r+1}$ in both members of (3.1) leads immediately to:

THEOREM 1. For $p$ odd $>1,0 \leq r \leq p$,

$$
\begin{array}{r}
\left(\begin{array}{c}
p+1 \\
r
\end{array}\right) k^{r}(c(h, k)-h)^{p+1-r}=\left(\begin{array}{c}
p+1 \\
r+1
\end{array}\right) h^{p-r}(c(k, h)-k)^{r+1} \\
+k B_{p+1-r} B_{r}-h B_{p-r} B_{r+1}
\end{array}
$$

In the next place, if for brevity we put $w=k \tau-h$, then (3.1) becomes

$$
\begin{aligned}
& k^{p}(w+h)(w+c(h, k))^{p+1} \\
& \quad=((w+h) c(k, h)-w k)^{p+1}+w(B k+(w+h) B)^{p+1}
\end{aligned}
$$

We now compare coefficients of $w^{r+1}$ in both members of (3.3); a little care is required in connection with the extreme right member. We state the result as:

THEOREM 2. For $p$ odd $>1,0 \leq r \leq p$,

$$
\left(\begin{array}{c}
p+1 \\
r+1
\end{array}\right) h k^{p} c_{p-r}(h, k)+\left(\begin{array}{c}
p+1 \\
r
\end{array}\right) k^{p} c_{p+1-r}(h, k)
$$




$$
=\left(\begin{array}{c}
p+1 \\
r+1
\end{array}\right) h^{p-r}(c(k, h)-k)^{r+1} c^{p-r}(k, h)+\left(\begin{array}{c}
p+1 \\
r
\end{array}\right)\left(B k+B^{\prime} h\right)^{p+1-r} B^{-r},
$$

where

$$
\left(B k+B^{\prime} h\right)^{p+1-r} B^{\prime r}=\sum_{s=0}^{p+1-r}\left(\begin{array}{c}
p+1-r \\
s
\end{array}\right) B_{p+1-r-s} B_{r+s} k^{p+1-r-s_{h} s} .
$$

For $r=0,(3.4)$ becomes

$$
\begin{aligned}
& (p+1) h k^{p} c_{p}(h, k)+k^{p} c_{p+1}(h, k) \\
& =(p+1) h^{p}\left\{c_{p+1}(k, h)-k c_{p}(k, h)\right\}+(p+1)(B k+B h)^{p+1},
\end{aligned}
$$

which reduces to

$$
(p+1)\left\{h k^{p} c_{p}(h, k)+k^{p} h c_{p}(k, h)\right\}=(p+1)(B k+B h)^{p+1}+p B_{p+1} .
$$

This is Apostol's reciprocity theorem.

If we take $r=1$ in (3.4), we get

$$
\begin{aligned}
& p\left\{h^{2} k^{p} c_{p-1}(h, k)-k^{2} h^{p} c_{p-1}(k, h)\right\} \\
& =-2\left\{h k^{p} c_{p}(h, k)+p k h^{p} c_{p}(h, k)\right\}+p B_{p+1}+2\left(B k+B^{\prime} h\right)^{p} B^{\prime} h .
\end{aligned}
$$

If in this formula we interchange $h$ and $k$ and add we again get (3.5), while if we subtract we get

$$
\begin{aligned}
p & \left\{h^{2} k^{p} c_{p-1}(h, k)-k^{2} h^{p} c_{p-1}(k, h)\right\} \\
& =(p-1)\left\{h k^{p} c_{p}(h, k)-k h^{p} c_{p}(k, h)\right\}-(B k+B h)^{p}(B k-B h) .
\end{aligned}
$$

In view of (3.6), it does not seem likely that Theorem 2 will yield a simple expression for

$$
h^{r+1} k^{p} c_{p-r}(h, k)+(-1)^{r} k^{r+1} h^{p} c_{p-r}(k, h) \quad(r>0) .
$$

We remark that Theorems 1 and 2 are equivalent. Indeed it is evident that 
(3.2) is equivalent to (3.1), and (3.4) is equivalent to (3.3); also it is clear that (3.1) and (3.3) are equivalent.

4. Some additional results. We next prove (compare $[6$, Th. 1]):

THEOREM 3. For $p, q \geq 1,0 \leq r \leq p+1$, we have

$$
c_{r}(q h, q k)=q^{r-p} c_{r}(h, k)
$$

Note that we now do not assume $p$ odd, $(h, k)=1$.

To prove (4.1), we have, using (1.2),

$$
\begin{aligned}
c_{r}(q h, q k) & =\sum_{\mu(\bmod q k)} P_{p+1-r}\left(\frac{\mu}{q k}\right) P_{r}\left(\frac{h \mu}{k}\right)_{\bullet} \\
& =\sum_{\substack{\nu(\bmod q) \\
\rho(\bmod k)}} P_{p+1-r}\left(\frac{\nu k+\rho}{q k}\right) P_{r}\left(\frac{h(\nu k+\rho)}{k}\right) \\
& =\sum_{\rho} P_{r}\left(\frac{h \rho}{k}\right) \sum_{\nu} P_{p+1-r}\left(\frac{\nu}{q}+\frac{\rho}{q k}\right) \\
& =q^{r-p} \sum_{\rho} P_{p+1-r}\left(\frac{\rho}{k}\right) P_{r}\left(\frac{h \rho}{k}\right) \\
& =q^{r-p} c_{r}(h, k) .
\end{aligned}
$$

For brevity we define

$$
b_{r}(h, k)=(c(h, k)-h)^{r}=\sum_{s=0}^{r}(-1)^{r-s}\left(\begin{array}{l}
r \\
s
\end{array}\right) h^{r-s} c_{s}(h, k),
$$

which occurs in Theorem 1. Clearly

$$
c_{r}(h, k)=(b(h, k)+h)^{r} \text {. }
$$

THEOREM 4. For $p, q \geq 1,0 \leq r \leq p+1$, we have

$$
b_{r}(q h, q k)=q^{r-p} b_{r}(h, k)
$$


By (4.1) and (4.2) we have

$$
\begin{aligned}
b_{r}(q h, q k) & =\sum_{s=0}^{r}(-1)^{r-s}\left(\begin{array}{l}
r \\
s
\end{array}\right)(q h)^{r-s} c_{s}(q h, q k) \\
& =\sum_{s=0}^{r}(-1)^{r-s}\left(\begin{array}{l}
r \\
s
\end{array}\right) h^{r-s} q^{r-p} c_{s}(h, k) \\
& =q^{r-p} b_{r}(h, k) .
\end{aligned}
$$

If we define

$$
a_{r}(h, k)=(c(h, k)-h)^{r} c^{p+1-r}(h, k),
$$

which is suggested by Theorem 2, we get:

THEOREM 5. For $p, q \geq 1,0 \leq r \leq p+1$,

$$
a_{r}(q h, q k)=q a_{r}(h, k) \text {. }
$$

The proof, which is exactly like the proof of (4.3), will be omitted.

We note that (4.4) implies

$$
h^{r} c^{p+1-r}(h, k)=\sum_{s=0}^{r}(-1)^{s}\left(\begin{array}{l}
r \\
s
\end{array}\right) a_{s}(h, k)=(1-a(h, k))^{r} .
$$

Also using (4.2) and (4.6), we get

$$
h^{p+1-r} b_{r}(h, k)=(1-a(h, k))^{p+1-r} a^{r}(h, k),
$$

and reciprocally from (4.4),

$$
a_{r}(h, k)=(b(h, k)+h)^{p+1-r} b^{r}(h, k) .
$$

Using $a_{r}(h, k)$ and $b_{r}(h, k)$, we can state Theorems 1 and 2 somewhat more compactly.

5. Another property of $c_{r}(h, k)$. For the next theorem compare [6, Th. 2].

THEOREм 6. For $p \geq 1,0 \leq r \leq p$, and $q$ prime, we have 
(5.1)

$$
\sum_{m=0}^{q-1} c_{r}(h+m k, q k)=\left(q+q^{1-p}\right) c_{r}(h, k)-q^{1-r} c_{r}(p h, k) .
$$

By (1.2), the left member of $(5.1)$ is equal to

$$
\begin{aligned}
\sum_{m=0}^{q-1} \sum_{\mu=1}^{q k} P_{p+1-r}\left(\frac{\mu}{q k}\right) P_{r}\left(\frac{(h+m k) \mu}{q k}\right) & \\
= & \sum_{\mu=1}^{q k} P_{p+1-r}\left(\frac{\mu}{q k}\right) \sum_{m=0}^{q-1} P_{r}\left(\frac{h \mu}{q k}+\frac{m \mu}{q}\right) \\
= & \sum_{\mu=1}^{q k} P_{p+1-r}\left(\frac{\mu}{q k}\right) P_{r}\left(\frac{h \mu}{k}\right) q^{1-r} \\
& \quad+\sum_{\nu=1}^{k} P_{p+1-r}\left(\frac{\nu}{k}\right)\left\{q P_{r}\left(\frac{h \nu}{k}\right)-P_{r}\left(\frac{q h \nu}{k}\right) q^{1-r}\right\} \\
= & q^{1-r} c_{r}(q h, q k)+q c_{r}(h, k)-q^{1-r} c_{r}(q h, k) \\
= & \left(q^{1-p}+q\right) c_{r}(h, k)-q^{1-r} c_{r}(q h, k),
\end{aligned}
$$

by (4.1).

It does not seem possible to frame a result like $(5.1)$ for the expressions $b_{r}(h, k)$ or $a_{r}(h, k)$ defined by $(4.2)$ and (4.3).

6. Representation by Eulerian numbers. If $k>1, \rho^{k}=1, \rho \neq 1$, we define the "Eulerian number" $H_{m}(\rho)$ by means of $[4, \mathrm{p} .825]$

$$
\frac{1-\rho}{e^{t}-\rho}=\sum_{m=0}^{\infty} H_{m}(\rho) \frac{t^{m}}{m !}
$$

Then it is easily verified that $[4, \mathrm{p} .825]$

$$
k^{m-1} \sum_{r=0}^{k-1} \rho^{r} B_{m}\left(\frac{r}{k}\right)=\frac{m}{\rho-1} H_{m-1}\left(\rho^{-1}\right),
$$

which may be put in the more convenient form 


$$
k^{m-1} \sum_{r(\bmod k)} \rho^{r} P_{m}\left(\frac{r}{k}\right)=\frac{m}{\rho-1} H_{m-1}\left(\rho^{-1}\right) .
$$

Now consider the representation (finite Fourier series)

$$
P_{m}\left(\frac{r}{k}\right)=\sum_{s=0}^{k-1} A_{s} \zeta^{-r s}
$$

If we multiply both members of $(6.3)$ by $\zeta^{r t}$ and sum, we get

$$
k A_{t}=\sum_{r} \zeta^{r t} P_{m}\left(\frac{r}{k}\right)= \begin{cases}\frac{m k^{1-m}}{\zeta^{t}-1} H_{m-1}\left(\zeta^{-t}\right) & (t \neq 0) \\ k^{1-m} B_{m} & (t=0)\end{cases}
$$

by (6.2) and (2.1). Thus (6.3) becomes

$$
P_{m}\left(\frac{\mu}{k}\right)=k^{-m} B_{m}+m k^{-m} \sum_{s=1}^{k-1} \frac{H_{m-1}\left(\zeta^{-s}\right)}{\zeta^{s}-1} \zeta^{-\mu s}
$$

Thus substituting from (6.4) in (1.2), we get after a little reduction

$$
c_{r}(h, k)=\frac{B_{p+1-r} B_{r}}{k^{p}}+\frac{r(p+1-r)}{k^{p}} \sum_{t=1}^{k-1} \frac{H_{p-r}\left(\zeta^{h t}\right) H_{r-1}\left(\zeta^{-t}\right)}{\left(\zeta^{-h t}-1\right)\left(\zeta^{t}-1\right)} .
$$

Thus $c_{r}(h, k)$ has been explicitly evaluated in terms of the Eulerian numbers. One or two special cases of (6.5) may be mentioned. For $r=p$ we have

$$
c_{p}(h, k)=\frac{p}{k^{p}} \sum_{t=1}^{k-1} \frac{H_{p-1}\left(\zeta^{-t}\right)}{\left(\zeta^{-h t}-1\right)\left(\zeta^{t}-1\right)}
$$

while for $r=p=1$ we have

$$
\bar{s}(h, k)=\frac{1}{4 k}+\frac{1}{k} \sum_{t=1}^{k-1} \frac{1}{\left(\zeta^{-h t}-1\right)\left(\zeta^{t}-1\right)},
$$

where $\bar{s}(h, k)=c_{1}(h, k)$. Note that $\bar{s}(h, k)=s(h, k)+1 / 4$, where $s(h, k)$ is the ordinary Dedekind sum [6]. We also note that (6.4) becomes, for $m=1$, 


$$
P_{1}\left(\frac{\mu}{k}\right)=-\frac{1}{2 k}+\frac{1}{k} \sum_{s=1}^{k-1} \frac{\zeta^{-\mu s}}{\zeta^{s}-1}
$$

which is equivalent to a formula of Eisenstein.

Possibly (6.5) can be used to give a direct proof of Theorem 1 or Theorem 2 .

\section{REFERENCES}

1. T. M. Apostol, Generalized Dedekind sums and transformation formulae of certain Lambert series, Duke Math. J. 17 (1950), 147-157.

2. - Theorems on generalized Dedekind sums, Pacific J. Math. 2 (1952), $1-9$.

3. R. Dedekind, Erläuterungen zu zwei Fragmenten von Riemann, Gesammelte mathematische Werke, vol. 1, Braunschweig, 1930, pp.159-173.

4. G. Frobenius, Über die Bernoullischen Zahlen und die Eulerschen Polynome, Sitzungsber. Preuss. Akad. Wissenschaften (1910), 809-847.

5. H. Rademacher, Zur Theorie der Modulfunktionen, J. Reine Angew. Math. 167 (1932), $312-336$.

6. H. Rademacher and A. Whiteman, Theorems on Dedekind sums, Amer. J. Math. 63 (1941), $377-407$.

DUKE UNIVERSITY 


\section{PACIFIC JOURNAL OF MATHEMATICS}

\section{EDITORS}

\author{
R. M. BOEINSON \\ University of California \\ Berkeley 4, California \\ E. HewitT \\ University of Washington \\ Seattle 5 , Washington
}

\section{R. P. DILWOR TH}

California Institute of Technology

Pasadena 4, California

E. F. BECKENBACH

University of California

Los Angeles 24, California

\section{ASSOCIATE EDITORS}

$\begin{array}{ll}\text { H. BUSEMANN } & \text { P. R. HALMOS } \\ \text { HERBERT FFDERER } & \text { IIEINZ HOPF } \\ \text { MARSHALL IJALL } & \text { R. D. JAMES }\end{array}$

\author{
BøRGE JESSEN \\ PAUL LÉVY \\ GEORGE PÓLYA
}

\author{
J. J. STOKER \\ E. G. STR AUS \\ KÔSAKU YOSIDA
}

\section{SFONSORS}

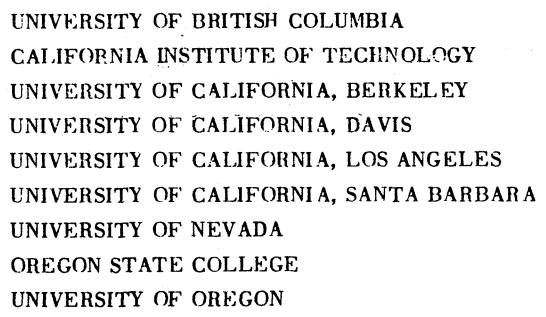

\author{
UNIVERSITY OF SOUTHERN CALIFORNIA \\ STANFORD RESEARCH INSTITUTE \\ STANFORD UNIVERSITY \\ WASHINGTON STATE COLLEGE \\ UNIVERSITY OF WASHINGTON \\ AMERICAN MATHEMATICAL SOCIETY \\ NATIONAL BUREAU OF STANDARDS, \\ INSTITUTE FOR NUMERICAL ANALYSIS
}

Mathematical papers intended for publication in the Pacific Journal of Mathematics should be typewritten (double spaced), and the author should keep a complete copy. Manuscripts may be sent to any of the editors except Robinson, whose term expires with the completion of the present volume; they might also be sent to M.M. Schiffer, Stanford University, Stanford, California, who is succeeding Robinson. All other communications to the editors should be addressed to the managing editor, E. F. Beckenbach, at the address given above.

Authors are entitled to receive 100 free reprints of their published papers and may obtain additional copies at cost.

The Pacific Journal of Mathematics is published quarterly, in March, June, September, and December. The price per volume (4 numbers) is $\$ 8.00$; single issues, $\$ 2.50$. Special price to individual faculty members of supporting institutions and to individual members of the American Mathematical Society: $\$ 4.00$ per volume; single issues, $\$ 1.25$.

Subscriptions, orders for back numbers, and changes of address should be sent to the publishers, University of California Press, Berkeley 4, California.

Printed at Ann Arbor, Michigan. Entered as second class matter at the Post Office, Berkeley, California.

\section{UNIVERSITY OF CALIFORNIA PRESS • BERKELEY AND LOS ANGELES}




\section{Pacific Journal of Mathematics}

\section{Vol. 3, No. 3 \\ May, 1953}

L. Carlitz, Some theorems on generalized Dedekind sums ............ 513

L. Carlitz, The reciprocity theorem for Dedekind sums ............. 523

Edward Richard Fadell, Identifications in singular homology theory..... . . 529

Harley M. Flanders, A method of general linear frames in Riemannian

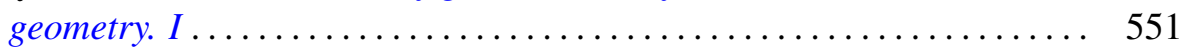

Watson Bryan Fulks, The Neumann problem for the heat equation ........ 567

Paul R. Garabedian, Orthogonal harmonic polynomials.............. 585

R. E. Greenwood and Andrew Mattei Gleason, Distribution of round-off errors for running averages .............................. 605

Arthur Eugene Livingston, The space $H^{p}, 0<p<1$, is not normable ... 613

M. N. Mikhail, On the order of the reciprocal set of a basic set of polynomials .......................................... 617

Louis Joel Mordell, On the linear independence of algebraic numbers . . . . 625

Leo Sario, Alternating method on arbitrary Riemann surfaces .......... 631

Harold Nathaniel Shapiro, Iterates of arithmetic functions and a property of the sequence of primes.............................. 647

H. Shniad, Convexity properties of integral means of analytic functions . . . . 657

Marlow C. Sholander, Plane geometries from convex plates ........... 667 